\title{
A study of the Hasegawa-Wakatani equations using an implicit explicit backward differentiation formula
}

\author{
L. Stals ${ }^{1}$
}

(Received 15 August 2008; revised 1 December 2008)

\begin{abstract}
The Hasegawa-Wakatani system of equations may be used to predict and study the behaviour of plasma flow. A recent analytical study of the use of linear multistep methods to solve the HasegawaWakatani equations showed the backward differentiation formulas to be the most stable. The backward differentiation formulas require a solution of a large dense system of equations, so we implemented an implicit explicit version of the formula. We study the performance of the implicit explicit backward differentiation formula on some example problems where the behaviour of the Hasegawa-Wakatani equation is predictable. These results suggest that the implicit explicit method is appropriate to use with the Hasegawa-Wakatani equations.
\end{abstract}

http://anziamj.austms.org.au/ojs/index.php/ANZIAMJ/article/view/1461 gives this article, (c) Austral. Mathematical Soc. 2008. Published December 16, 2008. ISSN 1446-8735. (Print two pages per sheet of paper.) 


\section{Contents}

1 Introduction

C520

2 The Hasegawa-Wakatani model

C521

2.1 Fourier spectral discretisation . . . . . . . . . . .

3 Calculating the energy equation

C523

4 Fortran code

C525

4.1 Model problem . . . . . . . . . . . . . . .

C525

$5 \quad$ Linear stability analysis

C527

5.1 Stable test problem . . . . . . . . . . . . . C527

5.2 Unstable test problem . . . . . . . . . . . . . . C530

6 Conclusion

C530

References

C532

\section{Introduction}

To design or predict the behavior of turbulent flows, prolonged time series are required so that meaningful statistics on patterns at various scales of motion are extracted. Studies over a large number of different parameters are also of interest to help better understand the behaviour of the parameter space. The computational challenge is to produce a code that generates these prolonged time series in a reasonable time on readily available machines.

In a recent work by Stals et al. [11] a comparative analysis of several classes of linear multistep methods was carried out in the context of optimising a code that simulates drift wave turbulence. The theoretical results 
obtained in the work were verified through prototype codes written in Scilab ${ }^{1}$.

A Fortran code based on an implicit explicit backward differentiation formula, specifically the SBDF4 method, has since been written and more substantial results have been obtained and are analysed in this report. In particular, we focus on the linearisation of the Hasegawa-Wakatani equations around certain equilibrium points where theoretical approximations are found to agree with the experimental results produced by the code.

\section{The Hasegawa-Wakatani model}

The Hasegawa-Wakatani model couples the flow field given by the electrostatic potential $\phi$ with the density $r$ [6]. The equations in a two dimensional domain are

$$
\begin{aligned}
\frac{\partial}{\partial t} \nabla^{2} \phi+\left[\phi, \nabla^{2} \phi\right] & =\alpha(\phi-r)-\beta_{\phi} \nabla^{6} \phi \\
\frac{\partial}{\partial t} r+[\phi, r] & =\alpha(\phi-r)-\kappa \frac{\partial \phi}{\partial y}-\beta_{r} \nabla^{4} r
\end{aligned}
$$

where $\phi=\phi(x, y, t), r=r(x, y, t)$, and $\nabla^{2 d}=\partial^{2 d} / \partial x^{2 d}+\partial^{2 d} / \partial y^{2 d}$ for $\mathrm{d}=1,2,3 . \nabla^{2}$ is the two dimensional Laplacian. The Poisson bracket [., .], defined by

$$
[f, g]=\frac{\partial f}{\partial x} \frac{\partial g}{\partial y}-\frac{\partial f}{\partial y} \frac{\partial g}{\partial x}
$$

gives the convective derivative. The parameters, $\alpha, \beta_{\phi}, \beta_{r}$ and $\kappa$ are all non-negative. The coefficients $\beta_{\phi}$ and $\beta_{r}$ denote viscosity and diffusion coefficients respectively. Physically, the $\mathrm{k}$ term feeds energy to the system, while the energy is dissipated by the parallel resistivity $(\alpha)$ and the hyperdiffusions.

Others discussed the physical interpretation of the system $[2,6,9,10]$.

${ }^{1}$ Scilab is freely available via http://www.scilab.org/ 


\subsection{Fourier spectral discretisation}

It is appropriate to use a Fourier spectral method to discretise Equations (1) and (2), since much of the essential physics of interest is contained in the energy distributions over the scales of the motions.

We assume that $\phi$ and $r$ are periodic over the domain $\Omega=[-\pi, \pi] \times$ $[-\pi, \pi]$. Following the structure given by Geveci [5] define

$$
\begin{aligned}
H^{6}(\Omega)= & \left\{u: u=\sum_{a, b=-\infty}^{\infty} u_{a, b} \omega_{x}^{a} \omega_{y}^{b}, \quad u_{a, b}^{*}=u_{-a,-b}, \quad u_{0,0}=0,\right. \\
& \left.\sum_{a, b=-\infty}^{\infty}\left(a^{2}+b^{2}\right)^{6}\left|u_{a, b}\right|^{2}<\infty\right\}
\end{aligned}
$$

where $\omega_{x}=e^{i x}, \omega_{y}=e^{i y}$, and $a$ and $b$ are integers. We assume that $\phi, r \in \mathrm{H}^{6}$. Set $\mathrm{m}=2 \mathrm{n}$ for some positive integer $n$ and let the corresponding discrete space $\mathrm{H}_{\mathrm{m}}^{6} \subset \mathrm{H}^{6}$ be defined as

$$
H_{m}^{6}(\Omega)=\left\{u: u \in H^{6}(\Omega), \quad u=\sum_{a, b=-n}^{n-1} u_{a, b} \omega_{x}^{a} \omega_{y}^{b}\right\} .
$$

Consequently $\phi_{\mathrm{m}} \in \mathrm{H}_{\mathrm{m}}$ and $\mathrm{r}_{\mathrm{m}} \in \mathrm{H}_{\mathrm{m}}$ are equivalent to the sums

$$
\phi_{m}(x, y)=\sum_{a, b=-n}^{n-1} \Phi_{a, b} \omega_{x}^{a} \omega_{y}^{b} \quad \text { and } \quad r_{m}(x, y)=\sum_{a, b=-n}^{n-1} R_{a, b} \omega_{x}^{a} \omega_{y}^{b} .
$$

Substituting expansion (4) into the Hasegawa-Wakatani equations (1) and (2), making use of the differentiation properties of the Fourier transform, and collecting like terms gives

$$
\frac{\partial}{\partial t} \Phi_{a, b}=\frac{\left(N\left(\phi, \nabla^{2} \phi\right)\right)_{a, b}}{\left(a^{2}+b^{2}\right)}-\frac{\alpha\left(\Phi_{a, b}-R_{a, b}\right)}{\left(a^{2}+b^{2}\right)}-\beta_{\phi}\left(a^{2}+b^{2}\right)^{2} \Phi_{a, b}
$$




$$
\begin{aligned}
\frac{\partial}{\partial t} R_{a, b}= & -(N(\phi, r))_{a, b}+\alpha\left(\Phi_{a, b}-R_{a, b}\right)-i b \kappa \Phi_{a, b} \\
& -\beta_{r}\left(a^{2}+b^{2}\right)^{2} R_{a, b},
\end{aligned}
$$

for all modes except the $\{0,0\}$ mode where $\Phi_{0,0}=R_{0,0}=0$.

Cover the real space domain $\Omega=[-\pi, \pi] \times[-\pi, \pi]$ by $(\mathrm{m}+1)^{2}$ uniformly spaced points $\left(x_{j}, y_{k}\right)$ where $-n \leq j, k<n$ ( $j$ and $k$ are integers).

The nonlinear terms $\left(\mathrm{N}\left(\phi, \nabla^{2} \phi\right)\right)$ and $(\mathrm{N}(\phi, r))$ are equivalent to a convolution function in the Fourier space. They are most efficiently calculated by firstly transforming them into the real space domain $\Omega$, evaluated as point-wise products, and then transforming the results back into the Fourier space $\mathrm{H}_{\mathrm{m}}^{6}$.

Let the vector $\boldsymbol{\phi} \in \mathbb{C}^{\mathrm{m} \times \mathfrak{m}}$ contain the potential on the grids $\phi_{j, k}=$ $\phi_{m}\left(x_{j}, y_{k}\right)$ where $-n \leq j, k<n$, and the vector $\Phi \in \mathbb{C}^{m \times m}$ contains the Fourier modes of the potential $\Phi_{a, b}$ where $-\mathfrak{n} \leq \mathrm{a}, \mathrm{b}<\mathrm{n}$. Similar relation holds for $\mathbf{r}, \mathbf{R} \in \mathbb{C}^{\mathrm{m} \times \mathrm{m}}$ where $\boldsymbol{r}$ contains the density on the grids $r_{j, k}=r\left(x_{j}, y_{k}\right)$ and $\mathbf{R}$ contains the Fourier modes $R_{a, b}$, respectively.

\section{Calculating the energy equation}

To perform a stability analysis of the Hasegawa-Wakatani equation an appropriate norm must be chosen. A discrete version of the energy equation given in previous studies $[2,9,10]$ worked well with the stability analysis presented by Stals et al. [11].

Define the discrete inner product between $\boldsymbol{x}, \mathbf{y} \in \mathbb{C}^{\mathfrak{m} \times \mathfrak{m}}\left(\boldsymbol{x}=\left(\boldsymbol{x}_{\mathfrak{j}, \mathrm{k}}\right)\right.$ and $\mathbf{y}=\left(y_{j, k}\right)$ with $\left.-\mathfrak{n} \leq j, k<n\right)$ as $\langle\boldsymbol{x}, \boldsymbol{y}\rangle_{2}=\sum_{j, k=-n}^{n-1} x_{j, k} y_{j, k}^{*}$ where ${ }^{*}$ denotes the complex conjugate. The corresponding norm is $\|x\|_{2}^{2}=\langle\boldsymbol{x}, \boldsymbol{x}\rangle_{2}$.

Let $\mathrm{D} \in \mathbb{R}^{\mathrm{m} \times \mathrm{m}}$ be defined by $\mathrm{D}_{\mathrm{a}, \mathrm{b}}=\mathrm{a}^{2}+\mathrm{b}^{2}(-\mathrm{n} \leq \mathrm{a}, \mathrm{b}<\mathrm{n})$. Also 
define $D_{n} \in \mathbb{R}^{m \times m}$ to be the same as $D$ except for the $\{0,0\}$ mode where $\left(D_{n}\right)_{0,0}=n$. All of the entries in $D_{n}$ are positive so $\langle\boldsymbol{x}, \boldsymbol{y}\rangle_{D_{n}}=\left\langle\boldsymbol{x}, D_{n} \cdot \boldsymbol{y}\right\rangle_{2}$ defines a norm. The '? in $\mathrm{D}_{n} \cdot \mathbf{y}$ represents point-wise multiplication.

We define the total system energy for the spectral equations (5) and (6) to be

$$
\mathcal{E}_{\mathrm{S}}^{2}=\frac{1}{2}\left(\|\boldsymbol{\Phi}\|_{\mathrm{D}_{\mathfrak{n}}}^{2}+\|\mathbf{R}\|_{2}^{2}\right)
$$

Then

$$
\frac{\partial \mathcal{E}_{S}^{2}}{\partial t}=\mathfrak{R}\left(-i \kappa\langle\Phi, B . R\rangle_{2}\right)-\left(\alpha\|\Phi-\mathbf{R}\|_{2}^{2}+\beta_{\phi}\|D \cdot \Phi\|_{D_{\mathfrak{n}}}^{2}+\beta_{\mathrm{r}}\|\mathrm{D} \cdot \mathbf{R}\|_{2}^{2}\right) .
$$

See previous work for a detailed explanation [11]. Observe that the nonlinear term does not appear in Equation (8). Stals et al. [11, Lemma 5.1] showed the nonlinear terms drop out from the right-hand side.

When $\mathfrak{R}\left(-\mathfrak{i}<\langle\boldsymbol{\Phi}, \mathbf{B} . \mathbf{R}\rangle_{2}\right)$ is bounded, the Hasegawa-Wakatani equations is dissipative and the stability analysis of Hill [7] predicts the behaviour of linear multistep methods. Hill's work is an extension of G-stability introduced by Dahlquist $[3,4,8]$. An interesting conclusion from the analysis of Dahlquist and Hill is that much of our understanding of the behaviour of linear systems of ordinary differential equations also applies to nonlinear systems. We showed elsewhere [11] that the dissipative terms $\beta_{\phi} \nabla^{6} \phi$ and $\beta_{r} \nabla^{4} r$ in Equations (1) and (2) have the most influence on the stability of the system. The nonlinear terms are not expected to have much influence except for small values of $\beta_{\phi}$ and $\beta_{r}$.

Following the ideas presented by Pedersen et al. [10] we define

$$
E_{c}=\frac{\partial \mathcal{E}_{S}^{2}}{\partial t}+\Re\left(i_{k}\langle\Phi, B . R\rangle_{2}\right)+\alpha\|\Phi-R\|_{2}^{2}+\beta_{\phi}\|D \cdot \Phi\|_{D_{n}}^{2}+\beta_{r}\|D \cdot R\|_{2}^{2} .
$$

The time derivative in Equation (9) is approximated by using a fourth order difference equation and $E_{c}$ used as an estimate of the numerical error in examples where the exact solution is not known. 


\section{Fortran code}

To better understand the theoretical results presented in Section 3 a small prototype code was written in Scilab [11]. While Scilab is effective for testing a number of different numerical techniques it does not allow us to solve problems with large values of $\mathrm{m}$. Therefore a Fortran 90 code has been written to allow more realistic simulations.

The theoretical analysis showed that the backward differentiation formulas (BDF) method is the best linear multistep method for the HasegawaWakatani equations. The BDF method is an implicit method so a nonlinear system of equations must be solved at each time step. Unfortunately the Jacobian associated with the Hasegawa-Wakatani equations is a dense matrix of size $m^{2} \times m^{2}$ and while it is possible to obtain results for small test problems using Scilab another approach needed to be considered for larger problems.

Instead of the BDF method we implemented the SBDF4 method, which is a fourth order implicit explicit method. The SBDF 4 method combines an implicit method for the linear terms and an explicit method for the nonlinear terms. The analysis suggests this is a reasonable choice as the linear terms are expected to have the most influence on the stability.

\subsection{Model problem}

A simple model problem used to study the behaviour of the solvers is obtained by setting $\phi(x, y, t)=-\sin (x) \sin (y) e^{-t}$ and $r(x, y, t)=2 \sin (x) \sin (y) e^{-t}$. These functions are a solution to Equations (1) and (2) when $\boldsymbol{k}=0$ and $\beta=\beta_{\phi}=\beta_{r}=(2-3 \alpha) / 8$. We set $\alpha=0.5$. The start time is $t=0$ and end time is $t=1$ so the number of time steps is $h^{-1}$ where $h$ is the time step size. 


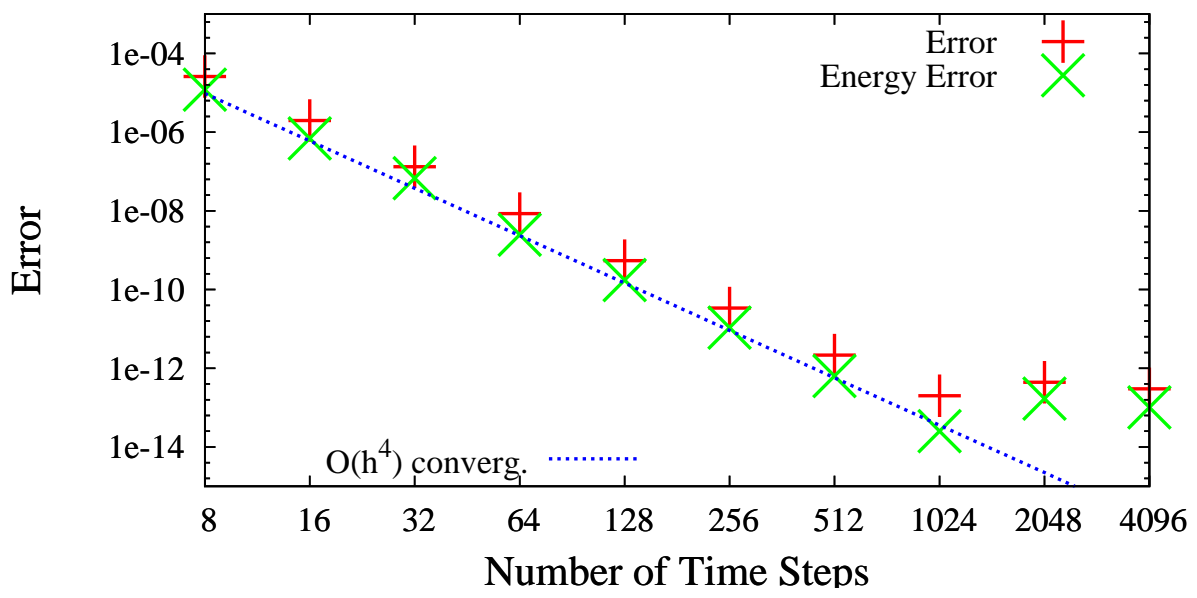

Figure 1: Convergence rate of $\boldsymbol{\Phi}$ for the SBDF4 method applied to the model problem. The number of time steps $=\mathrm{h}^{-1}$.

Algebraically the Poisson bracket should be zero for this example; however, due to round off it does not evaluate to exactly zero in the code.

Previous work [11] showed that the Adams-Bashforth and Adam-Moulton methods required small time step sizes before they showed convergence. The BDF method on the other hand worked well for large time steps. In those experiments $m$ was set to 32 . In all of the experiments for this article we increased $m$ to 256 .

The convergence rate for the SBDF 4 method is given in Figure 1. The results labelled Error are the difference between the calculated solution and the exact solution. The results labelled Energy Error are the numerical error estimate defined in Equation (9). The SBDF4 method shows the expected $\mathrm{O}\left(\mathrm{h}^{4}\right)$ convergence, and converged for large step sizes. 


\section{$5 \quad$ Linear stability analysis}

Camargo et al. [1] and Numata et al. [9] describe a linear stability analysis of the Hasegawa-Wakatani equations. We now use that analysis to further test our code and increase our understanding of the parameter space.

Assume that the solutions are of the form $e^{i(a x+b y-w t)}$ where $w=w_{r}+\mathfrak{i} w_{i}$ and $\boldsymbol{a}$ and $\boldsymbol{b}$ are the Fourier modes in Equation (4). Linearisation of the Hasegawa-Wakatani equations about the equilibrium point $\boldsymbol{\Phi}=\mathbf{R}=\mathbf{0}$ gives the dispersion relation

$$
w^{2}+i w\left(d+2 \beta k^{4}\right)-i d w_{*}-\alpha \beta k^{2}\left(k^{2}+1\right)=0,
$$

where $k^{2}=\left(a^{2}+b^{2}\right), d=\alpha\left(1+k^{2}\right) / k^{2}$ and $w_{*}=b k /\left(1+k^{2}\right)[1,9]$. Solving for $w$ gives

$$
w_{i}=-\frac{d+2 \beta k^{4}}{2} \pm A \sqrt{C+1} \quad \text { and } \quad w_{r}= \pm A \sqrt{C-1}
$$

where $A=d /(2 \sqrt{2})$ and $C=\sqrt{1+16 w_{*}^{2} / d^{2}}$. The system is stable when $w_{i} \leq 0$ and unstable when $w_{i}>0$. The results for a number of different parameter choices are shown in Figures 2 and 3 (we have not shown the results for varying $k$ due to space limitations). The thick, green line with crosses through it represents the curve where $w_{i}=0$. The region to the left of the thick line is where $w_{i}>0$ and the modes in that region are expected to be unstable. For example, the $\{1,1\}$ mode in all the tests shown in Figure 2 is unstable. The region to the right of the thick line indicates which modes are expected to be stable. The analysis suggests, for example, that increasing the dissipation parameters increases the stability of the system.

\subsection{Stable test problem}

In our first case study we set $\beta_{\phi}=\beta_{\mathrm{r}}=10^{-2}, \alpha=10^{-3}, \mathrm{k}=10^{-1}$ and $\mathrm{m}=256$. The initial values are $\boldsymbol{\Phi}_{\mathrm{a}, \mathrm{b}}=10^{-4}$ for $1 \leq \mathrm{a}, \mathrm{b} \leq 10$, with 

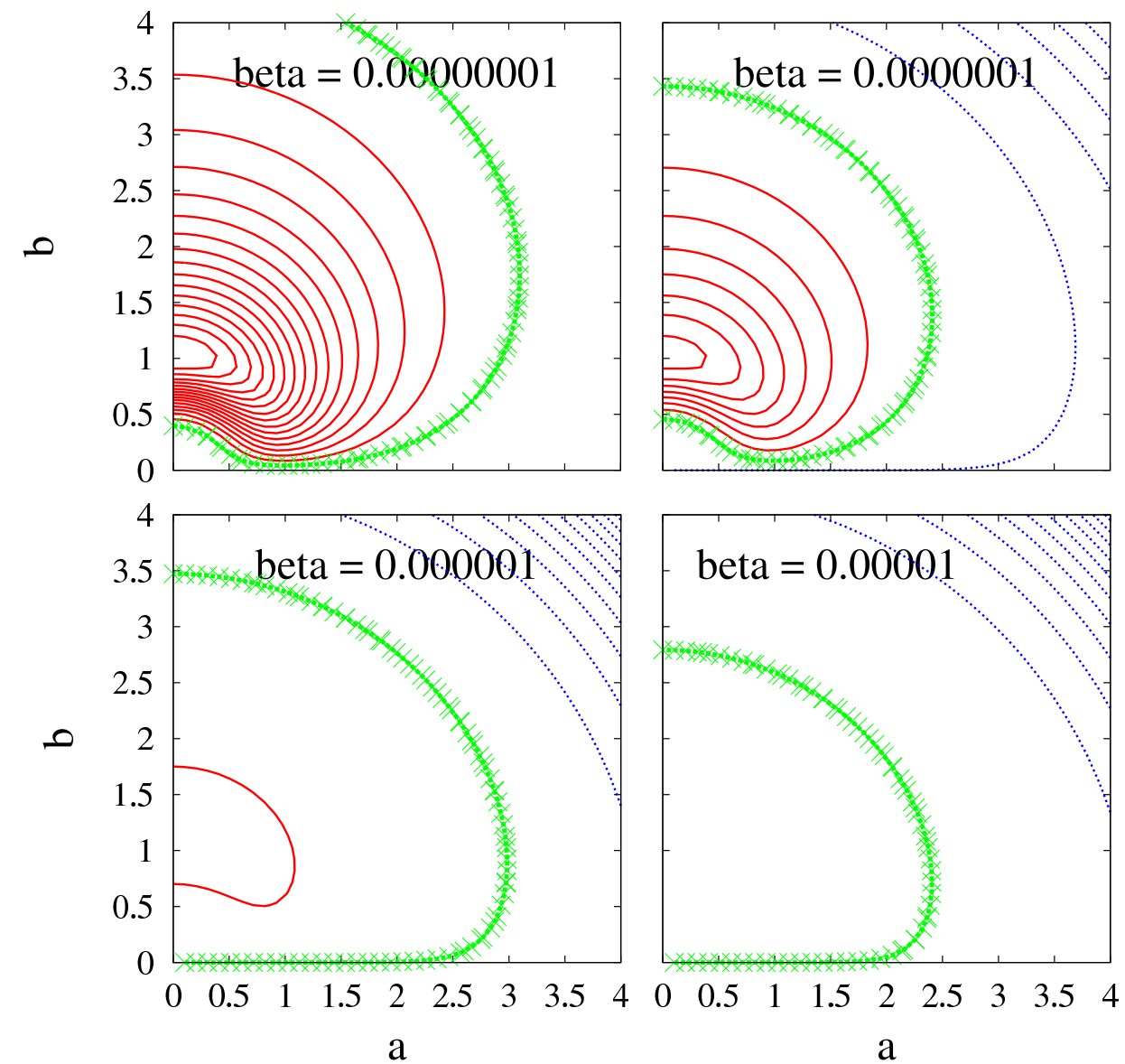

FiguRE 2: Linear stability analysis when $\alpha=\mathrm{k}=1.0$ and the dissipation coefficients $\beta=\beta_{\phi}=\beta_{\mathrm{r}}$ are varied. The plots are drawn for Fourier modes 0 to 4. 

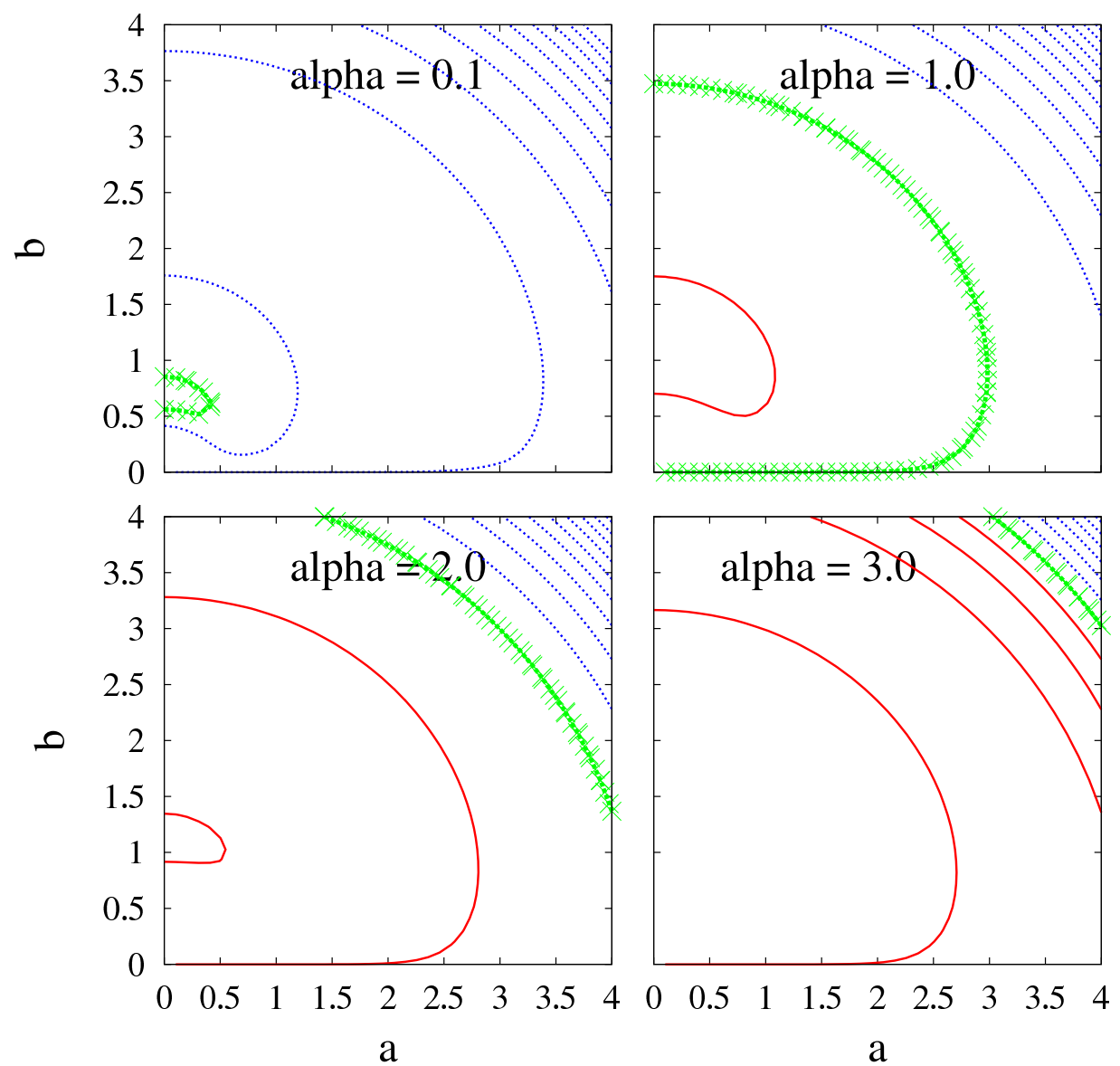

Figure 3: Linear stability analysis when $\beta=10^{-6}$ and $\kappa=1.0$, and the adiabaticity parameter $\alpha$ is varied. The plots are drawn for Fourier modes 0 to 4. 
$\boldsymbol{\Phi}_{-\mathrm{a},-\mathrm{b}}=\boldsymbol{\Phi}_{\mathrm{a}, \mathrm{b}}^{*}$, and all of the other modes were set to zero. The initial value of the density is the same as the potential, so $\mathbf{R}=\boldsymbol{\Phi}$. Based on the linear stability analysis we expect the system to be stable for all modes.

When running this test problem the high order modes are removed quickly while the low order modes are damped more slowly. This agrees with the linear stability analysis. The energy norm is reduced from $1.3 \times 10^{-7}$ at $t=5$ to $5.6 \times 10^{-8}$ at $t=20$ and $3.0 \times 10^{-8}$ at $t=40$ as the magnitude of the Fourier modes is reduced. The step size is $h=2.0 \times 10^{-2}$. The value of $E_{c}$ was below $2.2 \times 10^{-14}$ for all of the time steps.

\subsection{Unstable test problem}

In the next case study we set $\beta_{\phi}=\beta_{\mathrm{r}}=10^{-8}, \alpha=10^{\circ}, \mathrm{k}=5 \times 10^{-2}$ and $m=256$. The initial values are $\boldsymbol{\Phi}_{a, b}=10^{-4} i /\left(a^{2}+b^{2}\right)$ for $1 \leq a, b \leq 10$, all of the other modes were set to zero. Once again $\mathbf{R}=\boldsymbol{\Phi}$ at $\mathrm{t}=0$. According to the linear stability analysis the system will be unstable at modes $0 \leq a, b \leq 3$. The results showed an increase in the low order modes and consequently the energy norm increased as shown in Figure 4.

Table 1 lists $E_{c}$ for different values of $h$. The values of $E_{c}$ is roughly divided by 16 as $h$ is divided by two. We intend to use $E_{c}$ as an error indicator when introducing automatic time step selection. The solver did not converge for $h>2.44$ but it is still converging for relatively large time step sizes, which indicates that the SBDF4 method performs well on these example problems.

\section{Conclusion}

The SBDF4 method worked well on the example Hasegawa-Wakatani equations given in this report. Based on these results we plan to extend the 


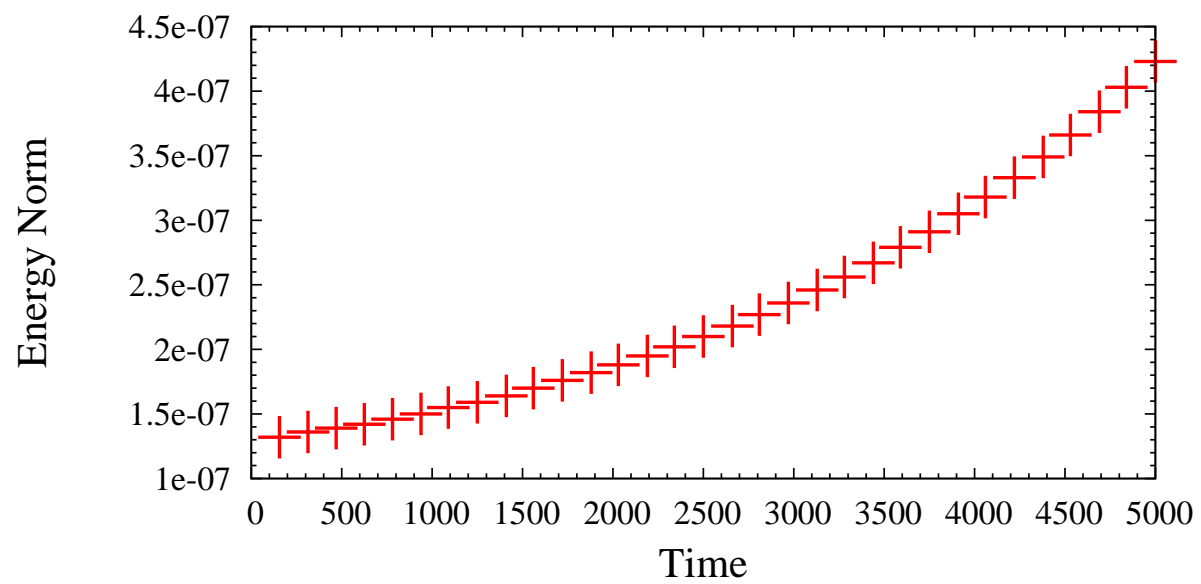

FiguRE 4: The energy norm increases as the magnitude of the Fourier modes increase in the unstable test problem.

TABLE 1: The energy conservation error $E_{c}$ at time $t=5000$ for the unstable test problem.

\begin{tabular}{cc}
\hline $\mathrm{h}$ & $\log \left(\mathrm{E}_{\mathrm{c}}\right)$ \\
\hline $2.44 \times 10^{0}$ & $1.38 \times 10^{-16}$ \\
$1.22 \times 10^{0}$ & $1.45 \times 10^{-17}$ \\
$6.10 \times 10^{-1}$ & $7.33 \times 10^{-19}$ \\
$3.05 \times 10^{-1}$ & $4.00 \times 10^{-20}$ \\
\hline
\end{tabular}


analysis presented by Stals et al. [11].

The complexity of the solutions to the Hasegawa-Wakatani equations varies considerably with the choice of parameters. We are working with $\mathrm{Nu}$ mata et al. [9] to develop a better understanding of the parameter space and nonlinear behaviour of the equations by using both theoretical and experimental results.

\section{References}

[1] S. J. Camargo, D. Biskamp, and B. D. Scott, Resistive drift-wave turbulence, Phys. Plasmas, 1, 1995, 48-62. http://www.Ideo. columbia.edu/ suzana/papers/camargo_biskamp_scott95.pdf C527

[2] S. J. Camargo, M. K. Tippett, and I. L. Caldas, Nonmodal energetics of resistive drift waves, Phys. Rev. E, 58, 1998, 3693-3704. doi:10.1103/PhysRevE.58.3693 C521, C523

[3] G. Dahlquist, On the relation of G-stability to other stability concepts for linear multistep methods, in Topics In Numerical Analysis III, J. H. Miller, ed., pages 67-80. Academic Press, London, 1977. C524

[4] G. Dahlquist, G-stability is equivalent to A-stability, BIT, 18, 1978, 384-401. doi:10.1007/BF01932018 C524

[5] T. Geveci, On the rate of convergence of the Fourier spectral method for the Navier-Stokes equations, Calcolo, 26, 1989, 185-195. doi:10.1007/BF02575728 C522

[6] A. Hasegawa and M. Wakatani, Plasma edge turbulence, Phys. Rev. Lett., 50, 1983, 682-686. doi:10.1103/PhysRevLett.50.682 C521 
[7] A. T. Hill, Global dissipativity for A-stable methods, SIAM J. Numer. Anal., 34, 1997, 119-142. doi:10.1137/S0036142994270971 C524

[8] R. LeVeque, G. Dahlquist, and D. Andree, Computations related to G-stability of linear multistep methods, Tech. Rep. STAN-CS-79-738, Stanford University, Computer Science Department, School of Humanities and Sciences, May 1979. ftp://reports.stanford.edu/ pub/cstr/reports/cs/tr/79/738/CS-TR-79-738.pdf C524

[9] R. Numata, R. Ball, and R. L. Dewar, Bifurcation in electrostatic resistive drift wave turbulence, Phys. Plasmas, 14, 102312, 2007, 8 Pages. http://arxiv.org/abs/0708. 4317 C521, C523, C527, C532

[10] T. S. Pedersen, P. K. Michelsen, and J. J. Rasmussen, Resistive coupling in drift wave turbulence, Plasma Phys. Control. Fusion, 38, 1996, 2143-2154. doi:10.1088/0741-3335/38/12/008 C521, C523, C524

[11] L. Stals, R. Numata, and R. Ball, Stability analysis of time stepping for prolonged plasma fluid simulations. Accepted for publication in SIAM Journal of Scientific Computing. C520, C523, C524, C525, C526, C532

\section{Author address}

1. L. Stals, Department of Mathematics, Australian National University, Australia.

mailto:stals@maths . anu . edu . au 